\title{
Chaotic Dynamics in Smart Grid and Suppression Scheme via Generalized Fuzzy Hyperbolic Model
}

\author{
Qiuye Sun, ${ }^{1}$ Yaguang Wang, ${ }^{1}$ Jun Yang, ${ }^{1}$ Yue Qiu, ${ }^{2}$ and Huaguang Zhang ${ }^{1}$ \\ ${ }^{1}$ College of Information Science and Engineering, Northeastern University, Shenyang 110004, China \\ ${ }^{2}$ Delft Center for System and Control, Delft University of Technology, Mekelweg 2, 2628 CD Delft, The Netherlands \\ Correspondence should be addressed to Jun Yang; yangjun@mail.neu.edu.cn
}

Received 9 April 2014; Revised 10 July 2014; Accepted 10 July 2014; Published 1 December 2014

Academic Editor: Evangelos J. Sapountzakis

Copyright (c) 2014 Qiuye Sun et al. This is an open access article distributed under the Creative Commons Attribution License, which permits unrestricted use, distribution, and reproduction in any medium, provided the original work is properly cited.

\begin{abstract}
This paper presents a method to control chaotic behavior of a typical Smart Grid based on generalized fuzzy hyperbolic model (GFHM). As more and more distributed generations (DG) are incorporated into the Smart Grid, the chaotic behavior occurs increasingly. To verify the behavior, a dynamic model which describes a power system with DG is presented firstly. Then, the simulation result shows that the power system can lead to chaos under certain initial conditions. Based on the universal approximation of GFHM, we confirm that the chaotic behavior could be suppressed by a new controller, which is designed by means of solving a linear matrix inequality (LMI). This approach could make a good application to suppress the chaos in Smart Grid. Finally, a numerical example is given to demonstrate the effectiveness of the proposed chaotic suppression strategy.
\end{abstract}

\section{Introduction}

Various types of stability problems in power systems, such as voltage collapse and oscillatory phenomenon, can be analyzed through chaotic theory [1]. Thus, chaos analysis has become an important analysis tool in stability studies of power networks. A chaotic system is a very special nonlinear dynamical system and it possesses several properties such as sensitivity to initial conditions, as well as an irregular, unpredictable behavior, and thereby confines the precise operation of the system. Such problems led to many severe events in the past $[2,3]$. Therefore, various effective methods have been proposed in the past decades to achieve the control and stabilization of chaotic systems, such as the LMI optimization approach [4], the nonfeedback method [5], inverse optimal control [6], the sliding method control [7], $H_{\infty}$ control [8, 9], impulsive control [10], backstepping design technique [11], cascade adaptive control [12], and the switching control [13]. However, although these references focus on restraining chaotic behavior, most of them are based on theoretical models and are not yet associated with power systems. Moreover, some previous articles which are devoted to chaos in power systems just consider traditional networks.
By contrast, they neglect the uncertainty caused by Smart Grid. The reason is that when distributed generation parallels in the grid, some system state changes [14-16].

Motivated by the aforementioned discussion, this study aims to present a control scheme to suppress chaos of a specific Smart Grid. To achieve this target, we innovatively come up with a dynamic model and then provide initial conditions which lead to the system falling into chaos. Then we establish a novel fuzzy model based on GFHM. In this model, we can simplify the task of assigning the performance of the closed-loop system. Finally, a novel controller is designed by solving a LMI to guarantee the stability of sliding motion and thereby achieve chaos suppression of the specific system.

The rest of the report is designed as follows. First of all, the fourth-order differential equation model of a typical Smart Grid is put forward via analytical modeling method where the wind turbine generator (WTG) is considered as the distributed source. Based on the established explicit mathematical model of the considered Smart Grid, when the system's parameters lie in a certain region, chaos is generated in the system, which yields greatly disadvantageous influences on the power grid. Secondly, by virtue of the 


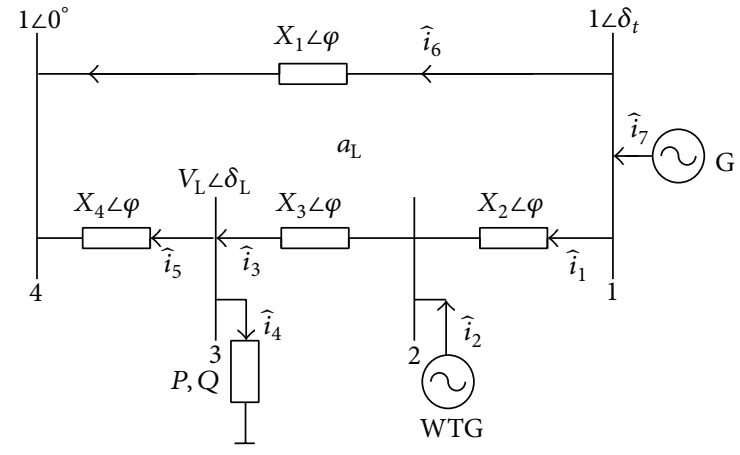

FIGURE 1: System infrastructure of Smart Grid.

universal approximation of GFHM, the chaotic system is approximated by a simple fuzzy model with prescribed precision. Then the sufficient conditions for the stabilization of the closed-loop system are derived based on a lemma and the controller gain matrices can be obtained by solving a LMI. In this way, a corresponding controller can be realized to suppress the chaotic behavior. Finally, a numerical example is given to demonstrate the effectiveness of the proposed chaotic suppression strategy.

\section{Chaotic Dynamics in Smart Grid}

2.1. System Model and Data. In order to analyze the electrical parameter variation, a typical infrastructure of Smart Grid has been examined in Figure 1. In the model, a power station is connected to an infinite Bus 1 through a purely inductive line. Bus 2 connects a WTG and there is a dynamic load at Bus 3. The system is connected to Bus 4.

Here, we suppose $X_{i}(i=1,2,3,4)$ is the corresponding impedance of the system and $\varphi$ is the angle of impedance. The voltage of infinite Bus 4 is $V_{1}$ and its phase is 0 . WTG works as a DG. It is a sixth-order model. Its model can be represented as follows [17]:

$$
\begin{gathered}
\dot{\delta}_{t}=\omega, \\
\dot{\omega}=\frac{-D_{m} \omega+T_{m}-T_{e}}{M},
\end{gathered}
$$

where $\delta_{t}$ is the rotator angle, $M$ is the generator inertia, $D_{m}$ is the damping factor per unit, $T_{m}$ is the generator input torque, and $T_{e}$ is the generator output torque.

The load at Bus 3 is chosen as the dynamic model:

$$
\begin{aligned}
& P=P_{0}+P_{1}+K_{1} \dot{\delta}_{\mathrm{L}}+K_{2} \dot{V}_{\mathrm{L}}+K_{3} V_{\mathrm{L}} \\
& \mathrm{Q}=\mathrm{Q}_{0}+\mathrm{Q}_{1}+K_{4} \dot{\delta}_{\mathrm{L}}+K_{5} V_{\mathrm{L}}+K_{6} V_{\mathrm{L}}^{2}
\end{aligned}
$$

The dynamic equations describing the system are conducted as follows. When we interconnect WTG with the power grid, the WTG should not influence the voltage of the connecting point. Therefore, the current will be the same before and after the WTG interconnection:

$$
\widehat{i}_{1}=\frac{1 \angle\left(\delta_{t}-\varphi\right)-V_{\mathrm{L}}\left(\delta_{\mathrm{L}}-\varphi\right)}{X_{2}+X_{3}} .
$$

The PWM inverter is used in this model to transform the interface circuit when we interconnect the WTG with the power grid [18]. According to the active power and reactive power, output of the PWM inverter is decoupled and thus they can be controlled, respectively. In this paper, we suppose that the output reactive power of WTG cancels the reactive power of Bus 1 at the interconnecting point. For the sake of convenience, the output active power current $i_{0}$ of PWM inverter is used to represent the output active power of the WTG. Consequently, the output current of PWM inverter is

$$
\widehat{i}_{2}=i_{0}+\frac{-j \sin \left(\delta_{t}-\varphi\right)+j V_{\mathrm{L}}\left(\delta_{\mathrm{L}}-\varphi\right)}{X_{2}+X_{3}} .
$$

The current flow through $X_{3}$ is

$$
\widehat{i_{3}}=\widehat{i}_{1}+\widehat{i}_{2}=i_{0}+\frac{\cos \left(\delta_{t}-\varphi\right)+j V_{\mathrm{L}}\left(\delta_{\mathrm{L}}-\varphi\right)}{X_{2}+X_{3}} .
$$

The current flow through $X_{4}$ is

$$
\hat{i}_{5}=\frac{V_{\mathrm{L}} \angle\left(\delta_{\mathrm{L}}-\varphi\right)-1 \angle(-\varphi)}{X_{4}} .
$$

The current at Bus 3 is

$$
\begin{gathered}
\hat{i}_{4}=\widehat{i}_{3}-\widehat{i}_{5}=i_{0}+\frac{\cos \left(\delta_{t}-\varphi\right)+j V_{\mathrm{L}} \angle\left(\delta_{\mathrm{L}}-\varphi\right)}{X_{2}+X_{3}} \\
-\frac{V_{\mathrm{L}} \angle\left(\delta_{\mathrm{L}}-\varphi\right)-1 \angle(-\varphi)}{X_{4}} .
\end{gathered}
$$

The power supplied at Bus 3 is

$$
\begin{aligned}
S= & V_{\mathrm{L}} \angle \delta_{\mathrm{L}} * \widehat{i}_{4}^{*} \\
= & V_{\mathrm{L}} i_{0} \angle \delta_{\mathrm{L}}+\frac{\cos \left(\delta_{t}-\varphi\right)-V_{\mathrm{L}} \cos \left(\delta_{\mathrm{L}}-\varphi\right)}{X_{1}+X_{2}} V_{\mathrm{L}} \angle \delta_{\mathrm{L}} \\
& -\frac{V_{\mathrm{L}}^{2} \angle \varphi-V_{\mathrm{L}} \angle\left(\delta_{\mathrm{L}}+\varphi\right)}{X_{4}} .
\end{aligned}
$$

The active power and reactive power at Bus 3 are

$$
\begin{aligned}
P= & i_{0} V_{\mathrm{L}} \cos \delta_{\mathrm{L}}+\frac{\cos \left(\delta_{t}-\varphi\right)-V_{\mathrm{L}} \cos \left(\delta_{\mathrm{L}}-\varphi\right)}{X_{1}+X_{2}} V_{\mathrm{L}} \cos \delta_{\mathrm{L}} \\
- & \frac{V_{\mathrm{L}}^{2} \cos \varphi-V_{\mathrm{L}} \cos \left(\delta_{\mathrm{L}}+\varphi\right)}{X_{4}} \\
Q= & i_{0} V_{\mathrm{L}} \sin \delta_{\mathrm{L}}+\frac{\cos \left(\delta_{t}-\varphi\right)-V_{\mathrm{L}} \cos \left(\delta_{\mathrm{L}}-\varphi\right)}{X_{1}+X_{2}} V_{\mathrm{L}} \sin \delta_{\mathrm{L}} \\
- & \frac{V_{\mathrm{L}}^{2} \sin \varphi-V_{\mathrm{L}} \sin \left(\delta_{\mathrm{L}}+\varphi\right)}{X_{4}}
\end{aligned}
$$


The deduced process of the active power for the power station is as follows:

$$
\begin{gathered}
\hat{i}_{6}=\frac{1 \angle\left(\delta_{t}-\varphi\right)-1 \angle(-\varphi)}{X_{1}}, \\
\hat{i}_{7}=\hat{i}_{1}+\hat{i}_{6} \\
=\left(\left(X_{1}+X_{2}+X_{3}\right) \angle\left(\delta_{t}-\varphi\right)-X_{1} V_{\mathrm{L}} \angle\left(\delta_{\mathrm{L}}-\varphi\right)\right. \\
\left.-\left(X_{2}+X_{3}\right) \angle(-\varphi)\right) \\
\times\left(X_{1}\left(X_{2}+X_{3}\right)\right)^{-1} .
\end{gathered}
$$

So the active power for the power station is

$$
P_{\mathrm{G}}=\operatorname{Re}\left\{1 \angle \delta_{t} * \hat{i}_{7}^{*}\right\},
$$

where $\operatorname{Re}\{\}$ represents the real part of $\{\bullet\}, \hat{i}_{7}^{*}$ represents the conjugate part of $\hat{i}_{7}$, and $P_{\mathrm{G}}$ is equal to the generator output torque $T_{e}$.

According to (11), the balance of active power and reactive power at Bus 3, and the system parameter values in Tables 1,2 , and $3[17,19]$, the system mathematic representation is derived as

$$
\begin{aligned}
\dot{\delta}_{t}= & \\
\dot{\omega}= & 0.573-0.167 \omega+20 V_{\mathrm{L}} \cos \left(\delta_{t}-\delta_{\mathrm{L}}+1.483\right) \\
& +11.667 V_{t} \cos \left(\delta_{t}+1.483\right) \\
\dot{\delta}_{\mathrm{L}}= & 69-93.33 V_{\mathrm{L}}-179.05 V_{\mathrm{L}}^{2}-50 i_{0} V_{\mathrm{L}} \sin \delta_{\mathrm{L}} \\
& -300 V_{\mathrm{L}} \sin \delta_{\mathrm{L}} \cos \left(\delta_{t}-1.483\right) \\
\dot{V}_{\mathrm{L}}= & 25.322 V_{\mathrm{L}}^{2}+13.054 V_{\mathrm{L}}+3.529 V_{\mathrm{L}} \cos \left(\delta_{t}-1.483\right) \\
& -3.529 V_{\mathrm{L}} \cos \delta_{\mathrm{L}} \cos \left(\delta_{\mathrm{L}}-1.483\right) \\
& +42.353 V_{\mathrm{L}} \sin \delta_{\mathrm{L}} \cos \left(\delta_{t}-1.483\right)+7.059 i_{0} V_{\mathrm{L}} \sin \delta_{\mathrm{L}} \\
& -42.353 V_{\mathrm{L}} \sin \delta_{\mathrm{L}} \cos \left(\delta_{\mathrm{L}}-1.483\right) \\
& -35.294 V_{\mathrm{L}} \sin \left(\delta_{\mathrm{L}}+1.483\right)+0.588 i_{0} V_{\mathrm{L}} \cos \delta_{\mathrm{L}} \\
& +2.941 V_{\mathrm{L}} \cos \left(\delta_{\mathrm{L}}-1.483\right)+1.31778 .
\end{aligned}
$$

2.2. Chaotic Behavior in Smart Grid. As we can see, the dynamics of (12) depend on five independent parameters, which makes a complete search in this large parameter space rather tedious. Since there is no simple method to predict which region of the parameter space is likely to yield chaotic solutions, some subsets $\left(\delta_{t}, \omega, \delta_{\mathrm{L}}, V_{\mathrm{L}}\right)^{T}$ of parameters are kept fixed while the inverter current $i_{0}$ is taken as a control parameter and allowed to vary arbitrarily.

Assume $x=\left(x_{1}, x_{2}, x_{3}, x_{4}\right)^{T}=\left(\delta_{t}, \omega, \delta_{\mathrm{L}}, V_{\mathrm{L}}\right)^{T}$. After searching several subsets $x$, we observed chaotic phenomenon when $x=(0.3,0.2,0.1,0.97)^{T}$ and $i_{0}=$ 0.01. Simulations of chaotic state response and Lyapunov
TABLE 1: Generator parameters (pu).

\begin{tabular}{lcc}
\hline$D_{m}$ & $M$ & $T_{m}$ \\
\hline 0.05 & 0.3 & 1 \\
\hline
\end{tabular}

TABLE 2: Network parameters (pu).

\begin{tabular}{lccccc}
\hline$X_{1}$ & $X_{2}$ & $X_{3}$ & $X_{4}$ & $\varphi$ & $\varphi$ \\
\hline 0.286 & 0.1 & 0.0667 & 0.2 & $85^{\circ}$ & $85^{\circ}$ \\
\hline
\end{tabular}

TABle 3: Dynamic load parameters (pu).

\begin{tabular}{cccccccccc}
\hline$K_{1}$ & $K_{2}$ & $K_{3}$ & $K_{4}$ & $K_{5}$ & $K_{6}$ & $P_{0}$ & $Q_{0}$ & $P_{1}$ & $Q_{1}$ \\
\hline 0.24 & 0.2 & 1.7 & -0.02 & -1.866 & 1.4 & 0.4 & 0.8 & 0 & 0.58 \\
\hline
\end{tabular}

exponents on this occasion are shown in Figures 2, 3, 4, 5, and 6. From these figures, we can see that as time elapses, $x$ does not return to its equilibrium state. On the contrary, it enters into a disorganized oscillation. This phenomenon proves that the system causes chaotic behavior. In addition, the positive Lyapunov exponent proves this conclusion.

\section{Controller Design Based on GFHM}

Aiming at systems with severe nonlinearity and strong coupling, such as the model considered here, the GFHM with universal approximation provides a useful approach to design a controller. We can utilize GFHM to approximate the system in arbitrary precision so as to provide a theoretical basis of controlling a strong nonlinear chaotic system. Furthermore, the corresponding controller based on GFHM can be designed via intelligent control theory. Therefore, the equivalent model of Smart Grid based on GFHM brings convenience to controller synthesis.

3.1. Review of a GFHM. In 2001, Zhang and Quan came up with a brand-new fuzzy model, which is the GFHM [20]. In this model, there are two types of fuzzy sets, including Positive $\left(P_{x}\right)$ and Negative $\left(N_{x}\right)$. The membership functions $P_{x}$ and $N_{x}$ are defined as

$$
\begin{aligned}
& u_{P_{x}}\left(x_{z}\right)=e^{-(1 / 2)\left(x_{z}-k_{z}\right)^{2}}, \\
& u_{N_{x}}\left(x_{z}\right)=e^{-(1 / 2)\left(x_{z}+k_{z}\right)^{2}},
\end{aligned}
$$

where $k_{z}>0$. However, only two fuzzy sets are not able to cover the whole input space. In order to make the model a universal approximator, we define new variables $\bar{x}_{i}$ by transforming the input variables $x_{z}$ as follows:

$$
\bar{x}_{i}=x_{z}-d_{i}
$$

where $i=1, \ldots, w(w$ is a positive integer $)$ and $d_{i}$ is a constant. We can see that, after the transformation of the input variables $x_{z}$, the fuzzy sets will cover the whole input space if $w$ is large enough.

Before defining the GFHM, the definition of generalized input variables and the generalized fuzzy hyperbolic rule base are given first. 


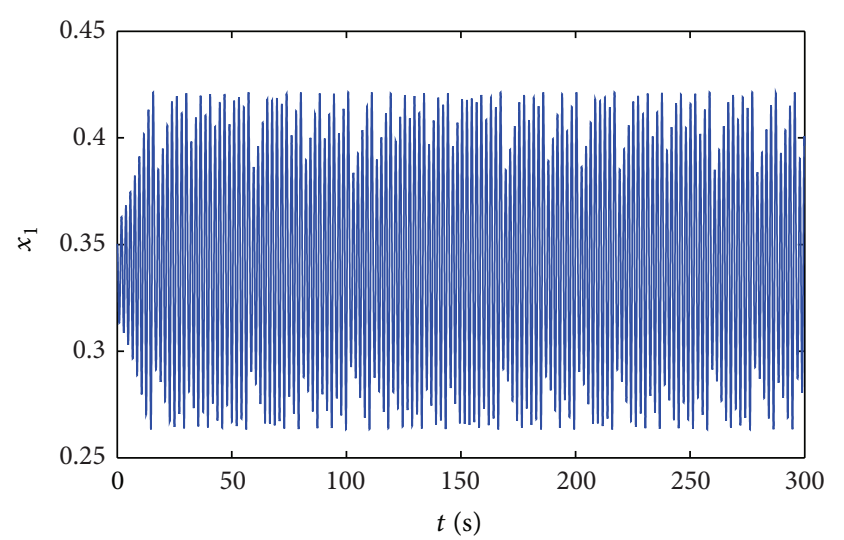

FIgURE 2: Chaotic state response curve of $x_{1}$.

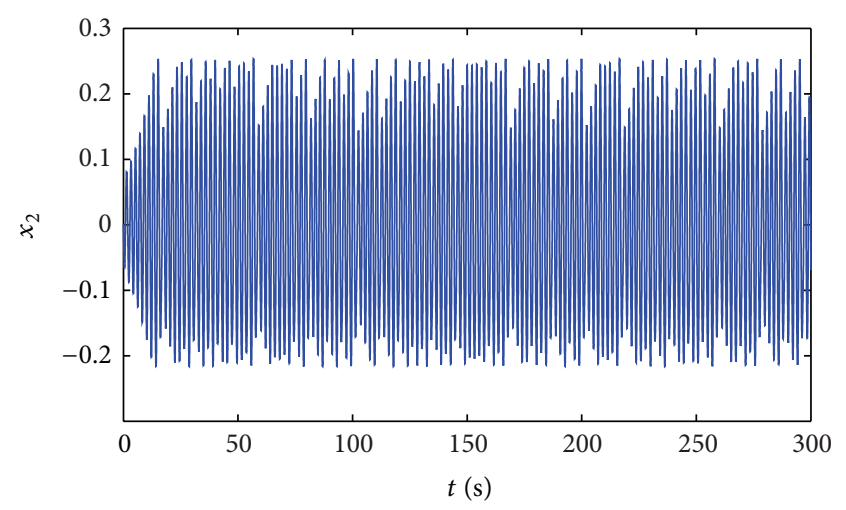

FIgUre 3: Chaotic state response curve of $x_{2}$.

Definition 1. Given a plant with $n$ input variables $x_{1}(t)$, $x_{2}(t), \ldots, x_{n}(t)$, the generalized input variables are defined as $\bar{x}_{1}=x_{1}-d_{11}, \ldots, \bar{x}_{w_{1}}=x_{1}-d_{1 w_{1}}, \ldots, \bar{x}_{w_{1}+1}=$ $x_{2}-d_{21}, \ldots, \bar{x}_{w_{1}+w_{2}}=x_{2}-d_{2 w_{2}}, \ldots, \bar{x}_{m-w_{n}+1}=x_{n}-$ $d_{n 1}, \ldots, \bar{x}_{m}=x_{n}-d_{n w_{n}}$, where $m=\sum_{i=1}^{n} w_{i}$ is the number of generalized input variables, $w_{z}(z=1, \ldots, n)$ represent the number of transformations associated with each $x_{z}$, and $d_{z j}\left(z=1, \ldots, n, j=1, \ldots, w_{z}\right)$ are constants that define the transformations.

Definition 2. Given a plant with $n$ input variables $x_{1}(t)$, $x_{2}(t), \ldots, x_{n}(t)$ and an output variable $\dot{x}$, the generalized input variables are defined as Definition 1 . And the fuzzy rule base is called the generalized fuzzy hyperbolic rule base if the following conditions are satisfied.

(1) The $l$ th fuzzy rule has the following form $(l=$ $\left.1,2 \ldots, 2^{m}\right)$ :

IF $\left(x_{1}-d_{11}\right)$ is $F_{x_{11}}, \ldots,\left(x_{1}-d_{1 w_{1}}\right)$ is $F_{x_{1 w_{1}}},\left(x_{2}-\right.$ $\left.d_{21}\right)$ is $F_{x_{21}} \ldots,\left(x_{n}-d_{n 1}\right)$ is $F_{x_{n} 1}$, and $\left(x_{n}-d_{n w_{n}}\right)$ is $F_{x_{n} w_{n}}$, THEN $\dot{x}^{l}=c_{F_{11}}+\cdots+c_{F_{1 w_{1}}}+c_{F_{21}}+\cdots+$ $c_{F_{2 w_{2}}}+\cdots+c_{F_{n w_{n}}}$, where $w_{z}(z=1, \ldots, n)$ represent the number of transformations associated with each $x_{z}, d_{z j}\left(z=1, \ldots, n, j=1, \ldots, w_{z}\right)$ are constants that define the transformations, $F_{x_{z j}}$ are fuzzy sets of

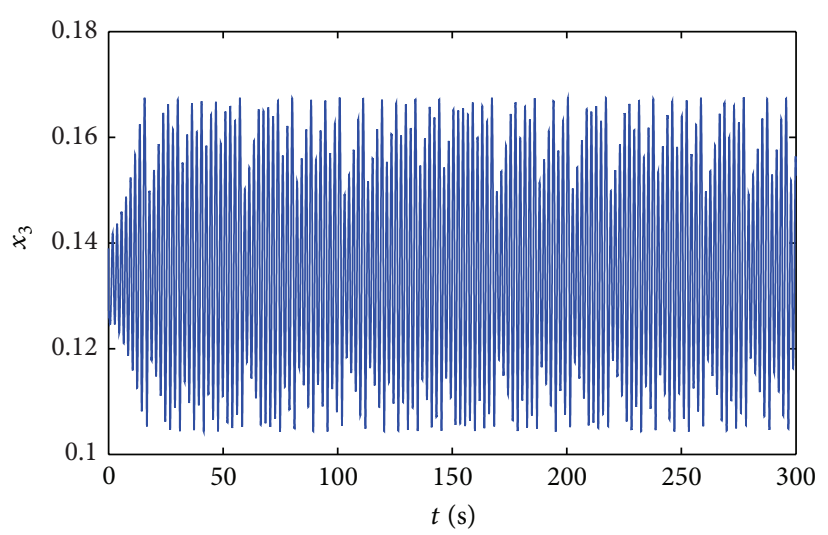

Figure 4: Chaotic state response curve of $x_{3}$.

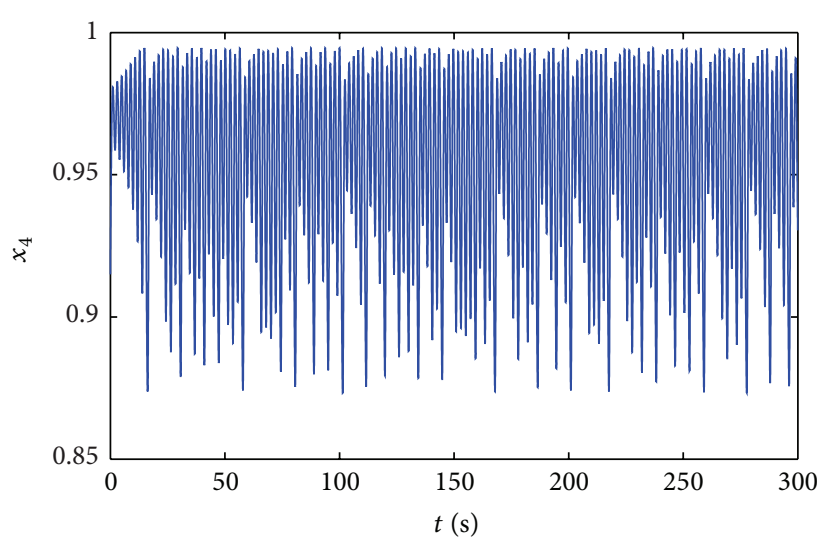

FIgURE 5: Chaotic state response curve of $x_{4}$.

$x_{z}-d_{z j}$ which include subsets $P_{x}$ and $N_{x}$, and $c_{F_{z j}}$ are constants corresponding to $F_{x_{z j}}$.

(2) The constants $c_{F_{z j}}\left(z=1, \ldots, n, j=1, \ldots, w_{z}\right)$ in the THEN-part correspond to $F_{x_{z j}}$ in the IF-part. That is, if there is $F_{x_{z j}}$ in the IF-part, $c_{F_{z j}}$ must appear in the THEN-part; otherwise, $c_{F_{z j}}$ does not appear in the THEN-part.

(3) There are $s=2^{m}$ fuzzy rules in the rule base, where $m=\sum_{i=1}^{n} w_{i}$, that is, all the possible $P_{x}$ and $N_{x}$ combinations of input variables in the IF-part and all the linear combinations of constants in the THENpart.

Lemma 3. For a plant with $n$ input variables $x_{1}(t), x_{2}(t), \ldots, x_{n}(t)$ and an output variable $\dot{x}$, if one defines the generalized fuzzy hyperbolic rule base and generalized input variables as Definition 2 and defines the membership function of the generalized input variables $P_{x}$ and $N_{x}$ as (13), then one can derive the following model:

$$
\dot{x}=A \tanh (K x),
$$




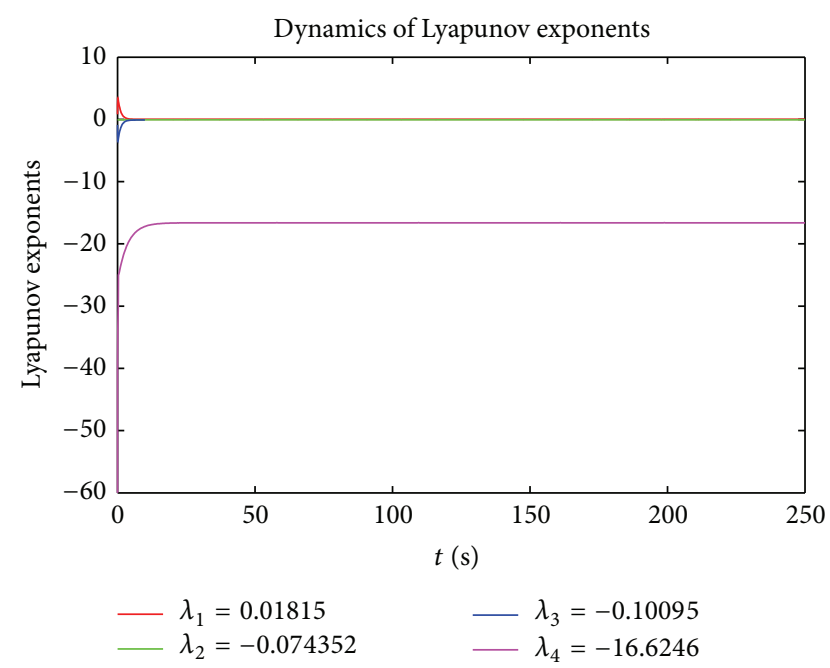

FIGURE 6: Lyapunov exponents.

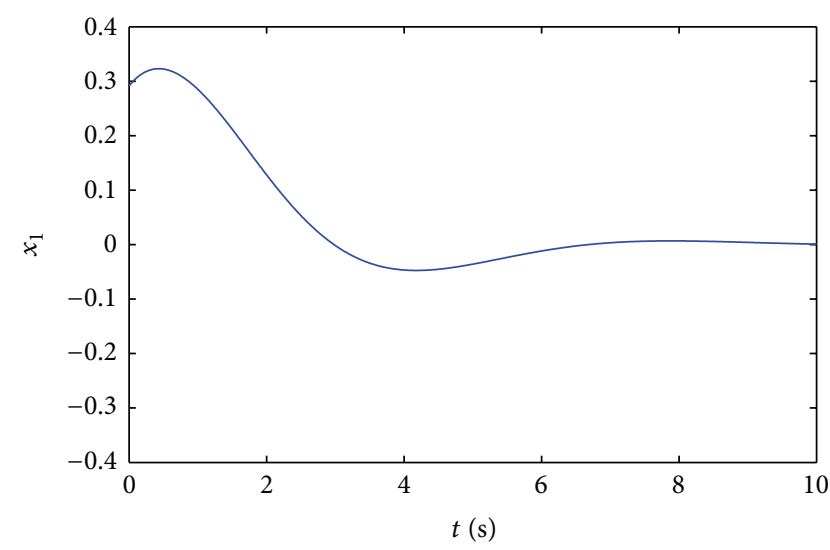

FIGURE 7: Stable state response curve of $x_{1}$.

where $\tanh (K x)=\left[\tanh \left(k_{1} x_{1}\right), \ldots, \tanh \left(k_{n} x_{n}\right)\right]^{T}$ and it is hyperbolic value of the state variables $x_{i}(i=1,2 \ldots n)$. One calls (15) as the GFHM.

Lemma 4. For any real continuous function $f$ defined in $U \subset$ $R^{n}$ and an arbitrary $\varepsilon>0$, there exists a system $g$ based on GFHM that makes $\sup _{x \in U}|f(x)-g(x)|<\varepsilon$.

Remark 5. Lemma 4 indicates that the GFHM has universal approximation, and thus the GFHM can be used to approximate the chaotic Smart Grid system in any precision theoretically. Therefore, we can stabilize the GFHM instead of the original strong nonlinearity system.

3.2. Chaotic Suppression Strategy. Now, we focus on designing a new controller, which stabilizes an equilibrium point of the closed-loop deterministic system for the corresponding chaotic system.

According to Lemma 4, the GFHM of Smart Grid can be established by parameter identification method [21]. Since it is significant to transform the equilibrium point to the origin point before establishing its GFHM, the GFHM of the chaotic Smart Grid system is expressed as

$$
\dot{x}=A \tanh x+i_{0} B \tanh x .
$$

$A$ and $B$ are obtained by matrix based on system identification:

$$
\begin{aligned}
& A=\left[\begin{array}{cccc}
0 & 1 & 0 & 0 \\
-1 & -0.95 & -7.17 & 12.32 \\
-25.46 & 0 & -10.98 & 287.64 \\
-22.98 & 0 & -214.84 & -150.34
\end{array}\right], \\
& B=\left[\begin{array}{cccc}
0 & 0 & 0 & 0 \\
0 & 0 & 0 & 0 \\
127.3 & 35.82 & 2.49 & 14.28 \\
114.9 & -61.6 & -378.28 & 32.34
\end{array}\right] .
\end{aligned}
$$

Therefore, the strong nonlinear system (12) can be represented as

$$
\dot{x}=\left(A+i_{0} B\right) \tanh x .
$$

The following theorem will give the advanced suppression strategy to the chaotic Smart Grid system.

Theorem 6. If there is a positive-definite diagonal matrix $P$ and a positive scalar $i_{0}$ such that the following LMI (19) holds, then the chaotic Smart Grid system can be stabilized asymptotically:

$$
P\left(A+i_{0} B\right)+\left(A+i_{0} B\right)^{T} P<0 .
$$

Proof. Denotethe Lyapunov function as

$$
V(x)=2 \sum_{i=1}^{4} p_{i} \times \ln \left(\cosh x_{i}\right),
$$

where $p_{i}>0(i=1,2,3,4)$, and then we can get

$$
\dot{V}(x)=2(\tanh x)^{T} P \dot{x},
$$

where $P=\operatorname{diag}\left(p_{1}, p_{2}, p_{3}, p_{4}\right)>0$.

Combining system (18), we can obtain

$$
\dot{V}(x)=(\tanh x)^{T}\left(P\left(A+i_{0} B\right)+\left(A+i_{0} B\right)^{T} P\right)(\tanh x),
$$

where $i_{0}$ is the output active power current of the PWM inverter. Hence, when the LMI (19) holds, (22) is negative. So the system (18) can be stabilized asymptotically.

Remark 7. In the above theorem, the stabilization condition for a closed-loop chaotic Smart Grid system is obtained based on the GFHM. Obviously, a new controller is designed by means of solving a LMI.

\section{Simulation Study}

With simulation study, we can illustrate the effectiveness of the proposed suppression scheme for (12). Here, we choose 


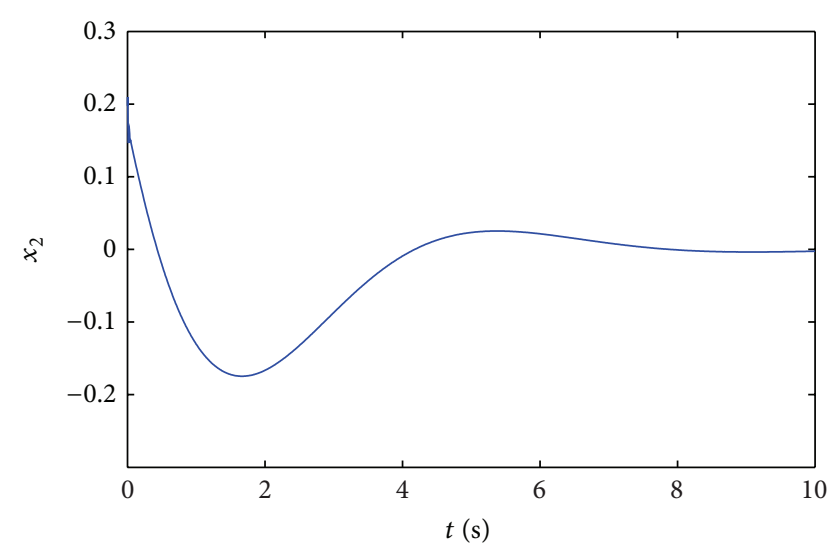

Figure 8: Stable state response curve of $x_{2}$.

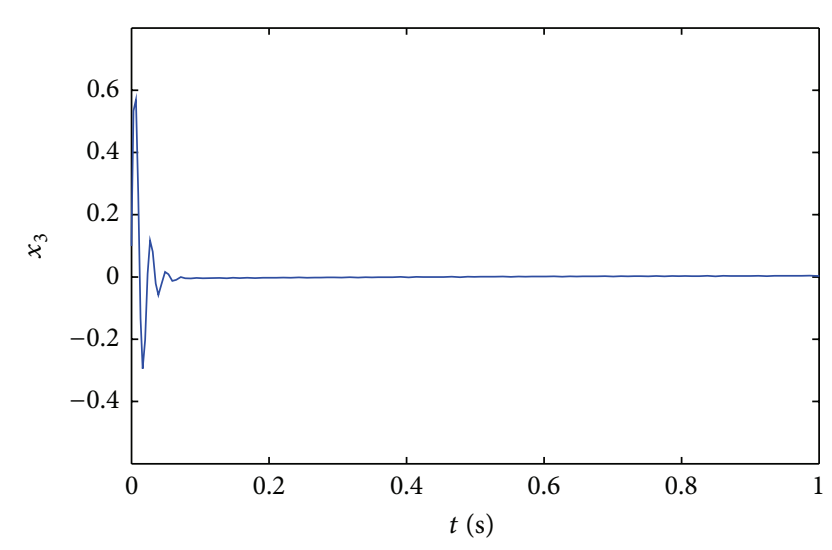

FIGURE 9: Stable state response curve of $x_{3}$.

the same initial condition in Section 2 and the matrix $P$ is chosen as a unit matrix. By solving (19), we can get $i_{0}=0.2$.

The state response curves of controlled system are shown in Figures 7, 8, 9, and 10 which, respectively, denote the stable state response curves $\left(x_{1}, x_{2}, x_{3}, x_{4}\right)$. Since we have observed chaotic phenomenon before when $x=\left(x_{1}, x_{2}, x_{3}, x_{4}\right)^{T}=$ $\left(\delta_{t}, \omega, \delta_{\mathrm{L}}, V_{\mathrm{L}}\right)^{T}=(0.3,0.2,0.1,0.97)^{T}$, the initial values of subsets $x$ are also set as $x=(0.3,0.2,0.1,0.97)^{T}$ to demonstrate the effectiveness of the proposed suppression scheme based on generalized fuzzy hyperbolic model. From these figures, we can see that the state response curves $\left(x_{1}, x_{2}, x_{3}, x_{4}\right)$ of original controlled system return to the equilibrium state after several fluctuations. The simulation results show that the proposed controller can suppress chaos in the Smart Grid successfully.

\section{Conclusion}

In this paper, we have studied a typical Smart Grid with strong coupling and nonlinearity. In this research, it is shown that Smart Grid is more likely to fall into chaos, as more and more DG are incorporated. And a new controller is designed to suppress the chaos based on the GFHM, which will guarantee

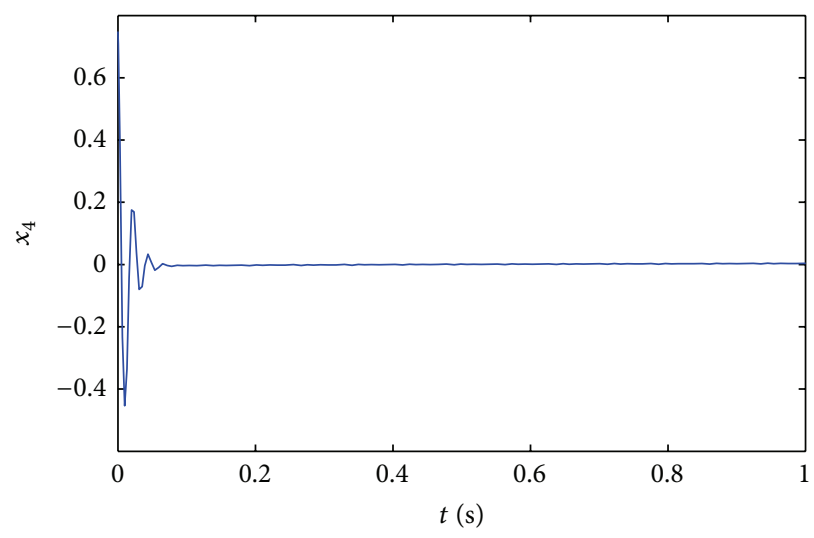

FIgURE 10: Stable state response curve of $x_{4}$.

that the power network is stable. The numerical simulation demonstrates its effectiveness.

\section{Conflict of Interests}

The authors declare that there is no conflict of interests regarding the publication of this paper.

\section{Acknowledgments}

Project was supported by the National Natural Science Foundation of China (Grants nos. 61104099 and 61374124), the Fundamental Research Funds for the Central Universities (Grants nos. N130404008 and N130404001), and the R\&D Project of State Grid Corporation of China (2013-0-1-04181).

\section{References}

[1] C. Cañizares, "Voltage stability assessment, procedures and guides," in IEEE/PES Power System Stability Subcommittee Special Publication, pp. 55-58, 2001.

[2] S. M. Amin and B. F. Wollenberg, "Toward a smart grid," IEEE Power and Energy Magazine, vol. 3, no. 5, pp. 34-41, 2005.

[3] W. Qiao, G. K. Venayagamoorthy, and R. G. Harley, "Missingsensor-fault-tolerantcControl for SSSC fACTS device with realtime implementation," IEEE Transactions on Power Delivery, vol. 24, no. 2, pp. 740-750, 2009.

[4] J. H. Park, O. M. Kwon, and S. M. Lee, "LMI optimization approach to stabilization of Genesio-Tesi chaotic system via dynamic controller," Applied Mathematics and Computation, vol. 196, no. 1, pp. 200-206, 2008.

[5] R. Li, W. Xu, and S. Li, "Chaos controlling of extended nonlinear Liénard system based on the Melnikov theory," Applied Mathematics and Computation, vol. 178, no. 2, pp. 405-414, 2006.

[6] Y. Alanis, E. A. Lastire, N. Arana-Daniel, and C. Lopez-Franco, "Inverse optimal control with speed gradient for a power electric system using a neural reduced model," Mathematical Problems in Engineering, vol. 2014, Article ID 514608, 21 pages, 2014.

[7] T. Y. Chiang, M. L. Hung, J. J. Yan, Y. S. Yan, and J. F. Chang, "Sliding mode control for uncertain unified chaotic systems with input nonlinearity," Chaos, Solitons and Fractals, vol. 34, no. 2, pp. 437-442, 2007. 
[8] J. H. Park, D. H. Ji, S. C. Won, and S. M. Lee, " $H_{\infty}$ synchronization of time-delayed chaotic systems," Applied Mathematics and Computation, vol. 204, no. 1, pp. 170-177, 2008.

[9] X. R. Liu, Q. Y. Sun, and X. M. Hou, "New approach on robust and reliable decentralized $H_{\infty}$ tracking control for fuzzy interconnected systems with time-varying delay," ISRN Applied Mathematics, vol. 2014, Article ID 705609, 11 pages, 2014.

[10] B. Wu, Y. Liu, and J. Q. Lu, "Impulsive control of chaotic systems and its applications in synchronization," Chinese Physics B, vol. 20, no. 5, Article ID 050508, 2011.

[11] E. Babaei, S. A. K. H. Mozaffari Niapour, and M. Tabarraie, "Design of a non-linear power system stabiliser using the concept of the feedback linearisation based on the back-stepping technique," IET Generation, Transmission \& Distribution, vol. 5, no. 8, pp. 860-868, 2011.

[12] W. Wei, D. H. Li, and J. Wang, "Cascade adaptive control of uncertain unified chaotic systems," Chinese Physics B, vol. 20, no. 4, Article ID 040510, 2011.

[13] J. Zhu, H. S. Xi, Q. Ling, and W. Xie, "Robust adaptive switching control for Markovian jump nonlinear systems via backstepping technique," Journal of Applied Mathematics, vol. 2012, Article ID 514504, 22 pages, 2012.

[14] H. Gholizadeh, A. Hassannia, and A. Azarfar, "Chaos detection and control in a typical power system," Chinese Physics B, vol. 22, no. 1, Article ID 010503, 2013.

[15] H. Chen, T. Lin, and J. Chen, "Dynamic analysis, controlling chaos and chaotification of a SMIB power system," Chaos, Solitons and Fractals, vol. 24, no. 5, pp. 1307-1315, 2005.

[16] H. Radmanesh and G. B. Gharehpetian, "Ferroresonance suppression in power transformers using chaos theory," International Journal of Electrical Power and Energy Systems, vol. 45, no. 1, pp. 1-9, 2013.

[17] K. G. Rajesh and K. R. Padiyar, "Bifurcation analysis of a three node power system with detailed models," International Journal of Electrical Power and Energy System, vol. 21, no. 5, pp. 375-393, 1999.

[18] Z. M. Ge, S. C. Li, and C. M. Chang, "Pragmatical adaptive chaos control from a new double van der Pol system to a new double Duffing system," Applied Mathematics and Computation, vol. 203, no. 2, pp. 513-522, 2008.

[19] I. Dobson and H. Chiang, "Towards a theory of voltage collapse in electric power systems," Systems \& Control Letters, vol. 13, no. 3, pp. 253-262, 1989.

[20] H. G. Zhang and Y. B. Quan, "Modeling, identification, and control of a class of nonlinear systems," IEEE Transactions on Fuzzy Systems, vol. 9, no. 2, pp. 349-354, 2001.

[21] Q. Sun, Y. Qiu, J. Zhou, J. Yu, and R. Zhang, "Observation of chaos in smart grid and stabilization condition via generalized fuzzy hyperbolic model," in Proceedings of the Asia-Pacific Power and Energy Engineering Conference, Wuhan, China, March 2011. 


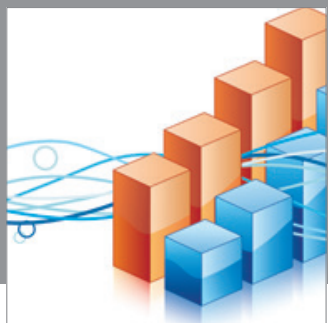

Advances in

Operations Research

mansans

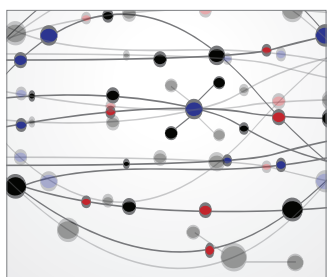

The Scientific World Journal
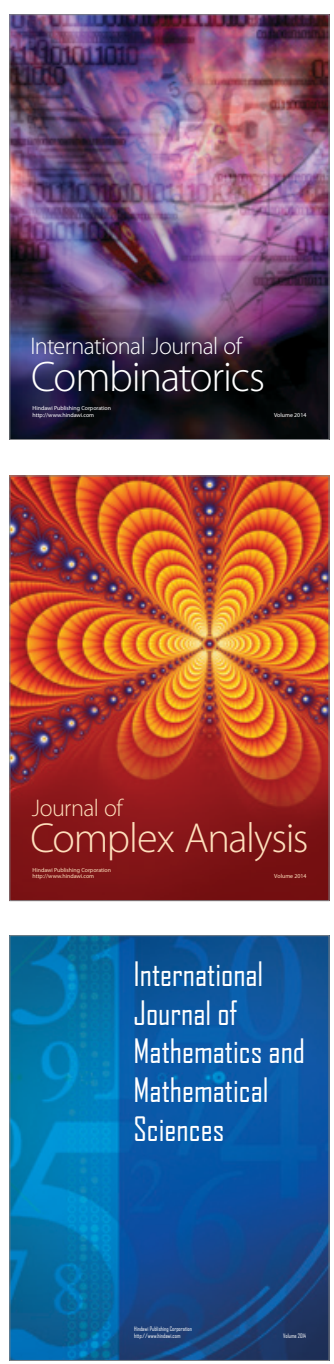
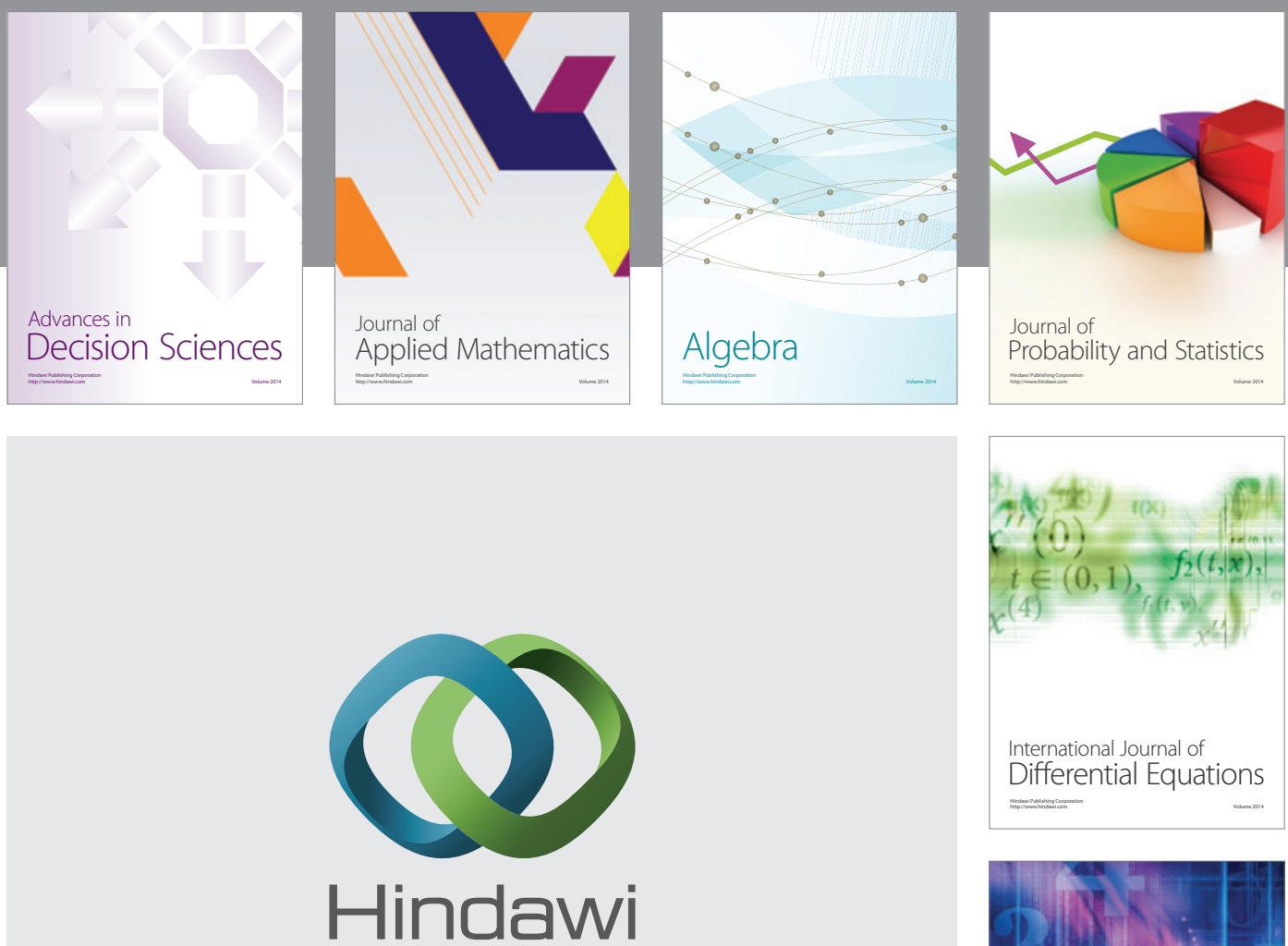

Submit your manuscripts at http://www.hindawi.com
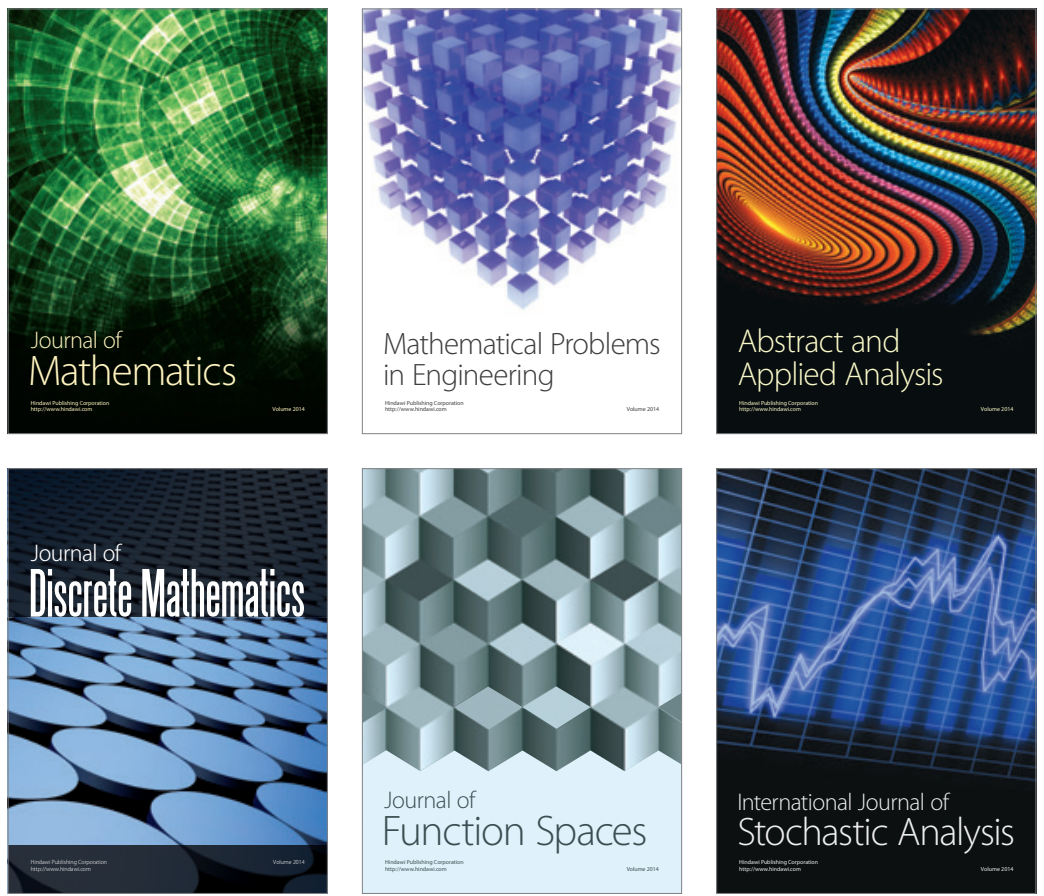

Journal of

Function Spaces

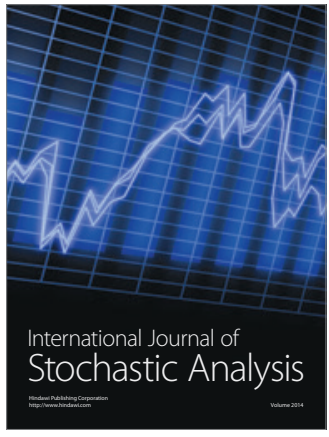

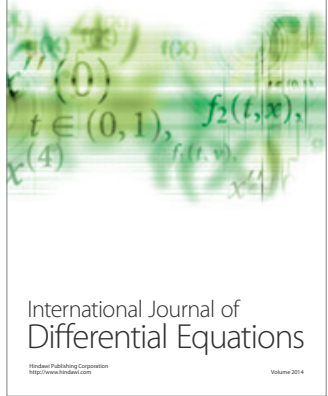
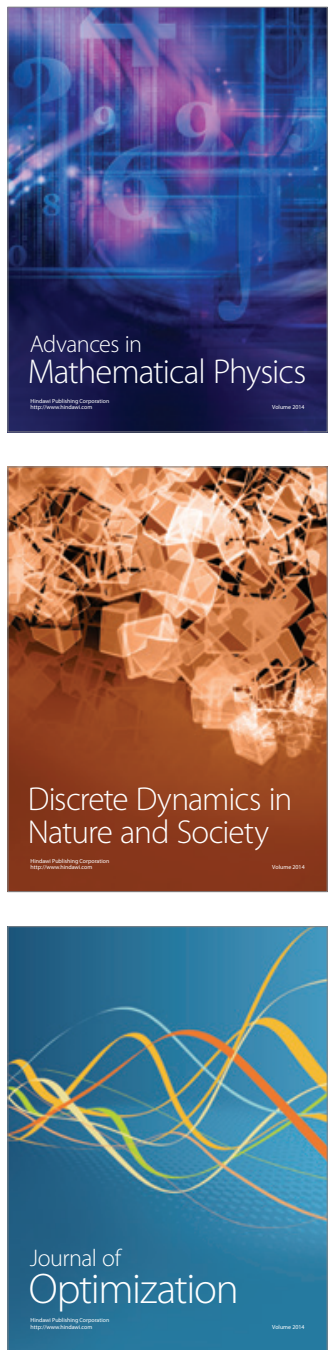